\title{
Wireless Monitoring System of Household Electrical Consumption with DALY-based Control Unit of Lighting Facilities Remotely Controlled by Internet
}

\author{
Paolo Visconti, Patrizio Primiceri, and Georgio Cavalera
}

\begin{abstract}
Aim of this paper is to present a wireless monitoring smart system of household electrical facilities, with ZigBee/WiFi transceivers, able to detect absorbed current from electrical loads, to calculate dissipated power and energy by means of PIC-based software and to view, in real-time, calculated consumption values on web page properly realized for user's remote control. Depending on light/presence sensors' signal, the realized system can switch on/off the monitored electrical loads for obtaining energy saving and user satisfaction. Also by sending DALI-standard commands to slave loads (e.g lighting facilities based on LEDs), the user can monitor, remotely by using a tablet/smartphone connected to internet, the operation's state and adjust the light intensity of each light point for achieving different scenarios. All functional tests, carried out on realized PCB prototype, have provided positive results allowing the use for the monitoring/driving of a real house's electrical facilities.
\end{abstract}

Index terms - DALI-protocol, Energy consumption minimization, Internet of Things, PCB design, Remote monitoring, ZigBee/WiFi wireless modules, Smart Home, Smart system.

\section{INTRODUCTION}

The energy issue has a leading position in the economic and development policies. In recent years, energy consumption have had a huge increase and to deal with these problems/needs two strategies were implemented: the intense use of renewable energy sources in place of non-renewable ones and the minimization/rationalization of energy consumption by using smart electronic systems of remote control and energy saving. The heart of a monitoring/energy saving system is a data acquisition/processing device installed on the photovoltaic or household electrical plant, in ongoing dialogue with sensors/actuators via serial or wireless communication. It supervises to ensure the highest efficiency and functionality with the possibility of communication to and from a supervision center located on plant (on site managing) or in remote location (remote control by Web) [1,2]. Remote control is performed by means of information displays located

Manuscript received September 27, 2015; revised February 26, 2016.

This work has been partially supported by Ministry of Education, University and Research (MIUR), Italy.

Authors are with the Department of Innovation Engineering (DII), University of Salento, Lecce, Italy and Cavalera S.r.l. Company, Galatone Italy (E-mails: paolo.visconti@unisalento.it, patrizio.primiceri@unisalento.it, g.cavalera@cavalerasrl.it). in local supervision center or through customized internet portals suitable to manage the production of any PV plant as well as energy consumption of household electrical facilities.

The plant's owner or technicians can access to data viewing's web site with simple and user-friendly interface at any time and in any place to monitor operation state and energy production/consumption data. In addition, the constant monitoring allows to promptly intervene in case of sudden malfunction or unjustified high consumption of the electrical loads $[3,4]$.

In this work, we have designed and developed a wireless monitoring system for the reduction of household energy waste. After careful research of wireless technology best suited to optimize system performance, we have realized and tested the hardware apparatus and the PIC-based software for managing and wireless remote control of home's electrical facilities based on both ZigBee and $\mathrm{WiFi}$ wireless technologies [3,5]. In experimental laboratory tests, we monitored in real time the energy consumption of lighting system, based on LEDs or low consumption bulbs, reporting the acquired data on web page properly designed for remote control. Many functions of household appliances or lighting facilities can be set from web page; for example pushing dedicated buttons you can turn on/off the lights or adjust their intensity [6-9]. Also the driving board was designed for assuming the master role in a network of lighting units driven by DALI protocol; therefore remotely by web page, the user can adjust the light intensity of each slave LED lamp for creating lighting scenarios $[10,11]$. In conclusion, by logging on to web page via a PC or tablet / smartphone, the user will monitor domestic energy consumption and regulate the operation of lighting systems for energy savings and user satisfaction for the better life's quality [12].

Semantically speaking, the Internet of Things can mean a world-wide network of interconnected objects uniquely addressable, based on standard communication protocols. This definition implies a tremendous number of objects, making part of everyone's life, capable of storing information, exchanging them and behaving according to them. In the wake of this explanation, another interesting definition is given by CISCO [13], where "IoT is simply the point in time when more things or objects were connected to the Internet than people", thus placing between 2008 and 2009, the birth of the 
Internet of Things. In [13], it is also stressed how the Internet of Things is the first real evolution of the common Internet: by making it sensory and aware of the physical world, it will allow to improve the way people live, making it easier and more efficient. IoT, in fact, will allow the creation of smart environments, thanks to the cooperation of several devices, which share data and are aware of the context in which they are used.

The birth of the Internet of Things, as described in [14], has been made possible thanks to many factors:

- The progressive miniaturization of sensors and chips, that makes easier the integration of several devices in a smaller area.

- The growth of wireless connectivity available, allowing the devices to create network in many circumstances.

- The use of cloud storage as a tool for sharing data and the development of sophisticated analytic models to extract information from them.

A huge flow of data can be generated by rethinking common objects with sensors and connection capability, creating an interacting network made by humans and things. In order to do that, however, it is important that those devices satisfy some requirements and specification: winning some of these challenges will contribute to increase the interest toward this technology and attract resources. Some of requirements that IoT devices should have are described below:

- Scalability: the main purpose of Internet of Things is putting several sensors on objects and connecting them. In order to handle the great amount of generated data, scalability in every layer of the network is required.

- Affordability: right now, the returns from investing in IoT are not enough to motivate the investors. For this reason, companies are trying to exploit mobile phones to collect data: these devices, used by the majority of population, are already full of sensors and always connected to Internet, thus providing a sort of first generation of applications for IoT. This "test bench" can be useful to show the potential of this technology and motivate the stakeholders.

- Context-awareness: the IoT vision will be eventually reached when it will be possible to derive a physical context through data aggregation coming from different devices. Knowing when, where and why something happened will make possible to react properly to different events; this feature implies also a huge development in automation processes.

- Cooperation: the development of applications should not be made in a vertical and independent way; instead, it would be better to share a common platform that promotes software re-use. However, since developing applications for IoT is a complex process requiring knowledge of different domains, some kind of cooperation among companies is necessary.

In this research context, we have developed a smart userconfigurable system for wireless managing and remotely control of household electric facilities based on ZigBee and $\mathrm{Wi}-\mathrm{Fi}$ wireless technologies.

\section{SYSTEM OPERATION IN THE MONITORING AND ENERGY SAVING OF HOUSEHOLD ELECTRICAL CONSUMPTION.}

The main function of realized monitoring system is the reduction/optimization of power consumption in the domestic environment. However as shown in figure 1, the monitoring and data transmitting unit can be used in other areas such as for wireless monitoring of PV plant's power production. In fact the designed board can be connected to ac outputs of PV plant's inverter and remotely accessing to web page by means of WiFi module, the user will monitor in real time PV energy production, consumption and energy needs of household appliances for obtaining better systems' integration and consequently higher energy savings.

Another application is to monitor real-time the energy consumption of one or more electrical loads (appliances, lighting units also driven by realized board) and their operation mode directly from PC or wirelessly via tablet or smartphone. In fact, you only need to be connected to home WiFi network and through a web browser to access the web page for adjusting the operation mode or simply viewing how much is consuming each of them (see figure 2).

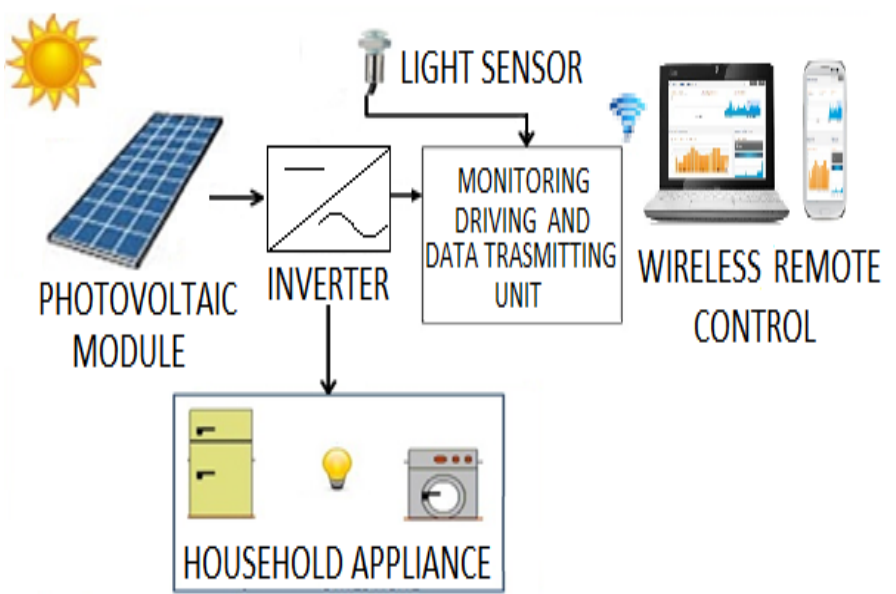

Fig. 1. Remote wireless monitoring, by means of tablet/smartphone connected to internet web page, of electrical energy produced from domestic PV plant and its integration with household appliances.

The designed system allows connection to itself by means of a WiFi wireless module; moreover the realized PCB prototype is planned for operation in a ZigBee network thanks to the use of a ZigBee transceiver based on 802.15.4 protocol, for assuming the gateway role in the ZigBee network [15-18].

In the realized system, five analog inputs are used to detect absorbed current from electrical loads for monitoring the instantaneous power consumption and calculating the used energy. Moreover to two PCB's digital inputs are connected presence and/or brightness sensors for avoiding energy waste in case of room lighting is not necessary because there aren't people inside or simply the natural daylight is sufficient (Figure 2). Another feature of designed system is the driving capability of new generation's lighting units, communicating with them by using DALI protocol; so the user, remotely connected to the web page, will monitor operating status, power on/off every light point and adjust their brightness level in order to achieve different scenarios or scenic effects. 
Regarding wireless communication between each monitoring/data transmitting unit and remote control station (see Figures 1 and 2), we have analyzed the technical features of wireless communication protocols (as shown in Table I); depending on technical and functional requirements of realized system, the advantageous parameters of each protocol for the specific application are reported of red color [15-18].

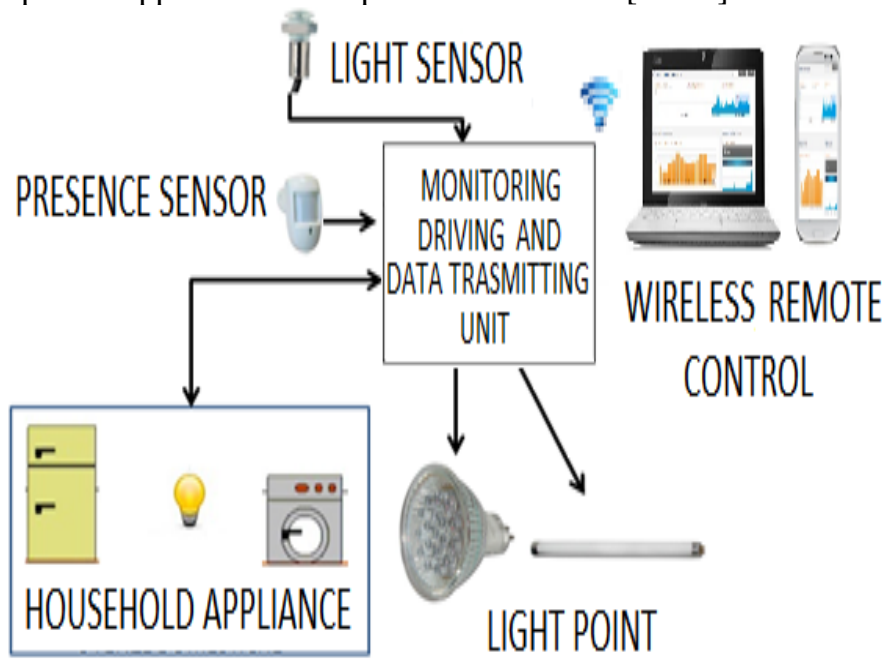

Fig. 2. Wireless monitoring of household electrical consumption and DALY-based ballast of lighting facilities remotely controlled by Internet.

TABLE I

SUMMARY OF TECHNICAL FEATURES RELATIVE TO WIRELESS COMMUNICATION'S PROTOCOLS (ZIGBEE, BLUETOOTH, WI-FI).

\begin{tabular}{|c|c|c|c|}
\hline $\begin{array}{l}\text { TECHNICAL } \\
\text { FEATURES }\end{array}$ & ZIG BEE & BLUETOOTH & WI-FI \\
\hline $\begin{array}{l}\text { WORKING } \\
\text { FREQUENCY }\end{array}$ & $\begin{array}{c}\text { 868/915 MHz; } \\
2.4 \mathrm{GHz}\end{array}$ & $2.4 \mathrm{GHz}$ & $\begin{array}{l}2.4 \mathrm{GHz} \\
5 \mathrm{GHz}\end{array}$ \\
\hline MAX DATA RATE & $20-250 \mathrm{~Kb} / \mathrm{s}$ & $1-3 \mathrm{Mb} / \mathrm{s}$ & $11-54 \mathrm{Mb} / \mathrm{s}$ \\
\hline $\begin{array}{c}\text { TRANSMISSION } \\
\text { DISTANCE }\end{array}$ & $10-100 \mathrm{~m}$ & $10 \mathrm{~m}$ & $10-100 \mathrm{~m}$ \\
\hline $\begin{array}{l}\text { NOMINAL TX } \\
\text { POWER }\end{array}$ & $\mid \begin{array}{l}-25 \text { - } 0 \mathrm{dBm} \\
\text { (VERY LOW) }\end{array}$ & $\begin{array}{l}\text { 0- } 10 \mathrm{dBm} \\
(\text { MEDIUM) }\end{array}$ & $\begin{array}{c}15-20 \mathrm{dBm} \\
(\text { HIGH) }\end{array}$ \\
\hline $\begin{array}{c}\text { NUMBER OF RF } \\
\text { CHANNELS }\end{array}$ & 16 & 79 & $\begin{array}{c}14(2.4 \mathrm{GHz}) \\
64(5 \mathrm{GHz})\end{array}$ \\
\hline $\begin{array}{c}\text { CHANNEL } \\
\text { BANDWIDTH }\end{array}$ & $\begin{array}{c}0.3 / 0.6 \mathrm{MHz} \\
2 \mathrm{MHz}\end{array}$ & $1 \mathrm{MHz}$ & 25-20 MHz \\
\hline MODULATION & $\begin{array}{c}\text { BPSK, QPSK, } \\
\text { O-QPSK }\end{array}$ & \begin{tabular}{|c|} 
GFSK, CPFS \\
8DPSK
\end{tabular} & $\begin{array}{l}\text { BPSK, M-QAM } \\
\text { QPSK, OFDM }\end{array}$ \\
\hline SPREADING & DSSS & FHSS & $\begin{array}{c}\text { MC-DSSS, } \\
\text { CCK, OFDM }\end{array}$ \\
\hline MAX N० NODES & $>65000$ & 8 & 2007 \\
\hline $\begin{array}{c}\text { DATA } \\
\text { PROTECTION }\end{array}$ & 16-bit CRC & 16-bit CRC & 32-bit CRC \\
\hline
\end{tabular}

By careful evaluation of each protocol's features, we have chosen to use the ZigBee communication mode between each monitoring/data transmitting unit due to high number of usable nodes/modules (>64000), the small data's amount to be transmitted in our specific application (max data rate $250 \mathrm{~Kb} / \mathrm{s}$ ) and above all for the reduced power consumption of ZigBee transceiver ensuring long lifetime of the supply battery. Therefore the designed system provides the use of a Zig Bee wireless network by means of Telegesis ETRX2 modules (for Gateway unit, reference and mobile nodes) with a server for internet access. The unique gateway module acts as coordinator, is fed permanently and charge of forwarding the data from the server to the sensors network and back.

Telegesis ETRX2 module, shown in figure $3 \mathrm{a}$, is a $2.4 \mathrm{GHz}$ transceiver based on EM250 single chip Ember with ZigBee/IEEE 802.15.4 protocol (Ref. 19 - Datasheet EM250 Single-Chip ZigBee/802.15.4 Solution). In figure $3 \mathrm{~b}$ are shown its block diagram with different building logic modules and the external pinout for easy access to embedded PIC (Ref. 20 - ETRX2 ZIGBEE module product manual). The ETRX2 device presents 12 general-purpose I/O lines and 2 analogue inputs; all 17 GPIO pins of EM250 chip with XAP2b PIC embedded are accessible. The communication with PIC is realized by serial UART interface through pins number 27-28.
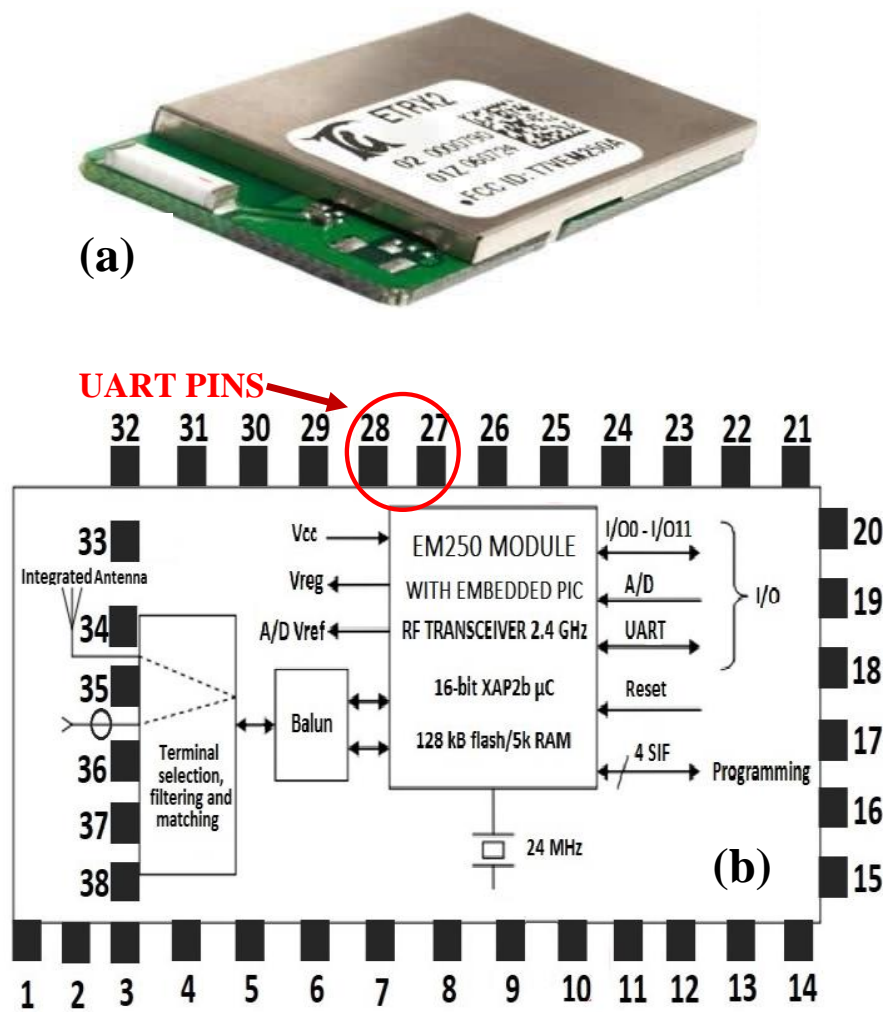

Fig. 3. Viewing of ETRX2 ZigBee Radio Module (a) and its block diagram with pinout description (b) for connecting itself with other electronic blocks.

\section{APPLICATIONS AND TECHNICAL FEATURES OF DALI COMMUNICATION SYSTEM}

DALI (Digital Addressable Lighting Interface) is a digital technology for management of lighting systems, as well as dimmerable LED-based facilities or other advanced devices, by using a digital addressing signal in order to univocally select up to 64 modules on a same data bus (Figure 4). Each module can belong to one or more of 16 available groups and 
can store 16 presets and various other parameters; all modules can talk to each other in bidirectional way since each of them has a unique address. For sending a command simultaneously to several modules, it is used the group address; in short, the sent commands on the DALI bus can be directed to a single lighting module, to a group (for achieving different scenarios) or to all connected modules (broadcast) [10,11].

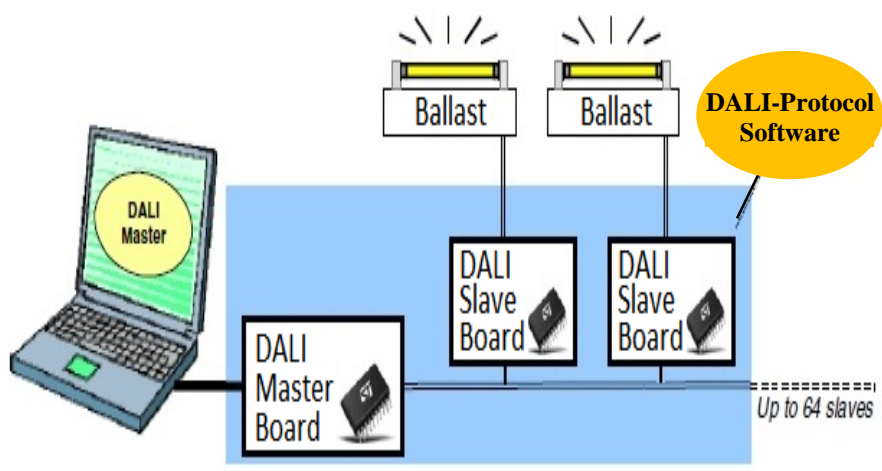

Fig. 4. Lighting facility based on DALI communication system with PC DALI Master and several DALI Slave Boards for managing each lamp's ballast.

DALI protocol can be used for managing light sources where is not required a high update rate of their operation so as for automatic adjustment of lamps' light intensity as function of natural light's contribution or room functionality.

The low logic level (active state) is defined with a voltage range between $-6.5 \mathrm{~V}$ and $+6.5 \mathrm{~V}$ while the high logic level (inactive state) with a voltage in the range $[+9.5 \mathrm{~V}-+22.5 \mathrm{~V}]$; consequently the voltage range $[+6.5 \mathrm{~V}-+9.5 \mathrm{~V}]$ is not defined (Figure 5).

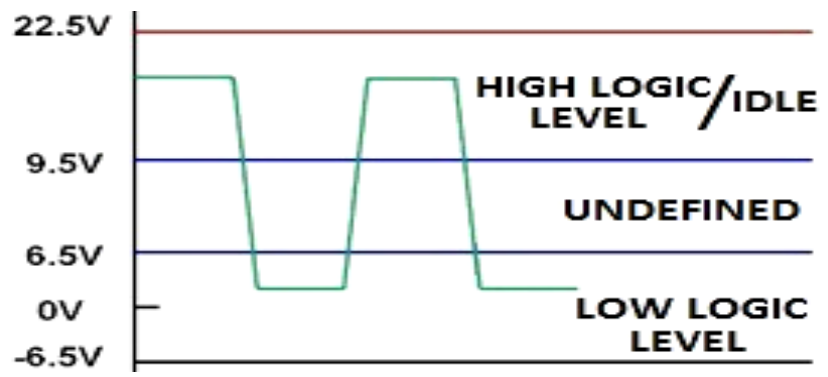

Fig. 5. Voltage range assigned to low and high logic levels for DALIbased communication system.
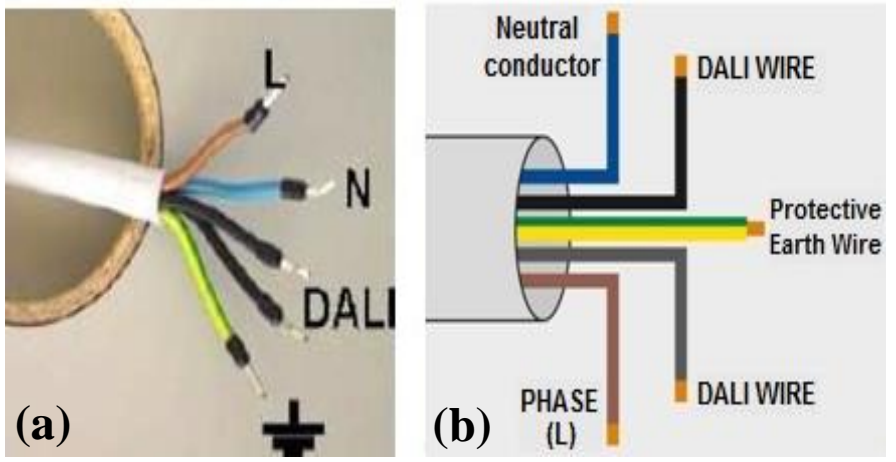

Fig. 6. View of real cable for DALI communication (a) and cable schematic with wires' specification (b).
In order to send the digital information strings on DALI bus (Figure 6) with data transfer rate equal to $1200 \mathrm{bit} / \mathrm{s}$, a serial protocol based on Manchester coding is used; so one logic level is represented by a falling edge (high level followed by low level) and zero logic level by a rising edge (low level followed by high level). The high and low pulses have the same time duration $\mathrm{Te}=416 \mu \mathrm{S}$ equal to half of digital signal's period $2 \mathrm{Te}=833 \mu \mathrm{S}$.

\section{BLOCK DIAGRAM OF REALIZED WIRELESS MONITORING SYSTEM WITH DALY-BASED CONTROL UNIT}

The heart of designed electronic system is the Open Picus FlyPort WiFi module chosen after long and careful research having, related to project idea to be implemented, the required and necessary technical features and also being cheaper and simpler to use respect to other similar development boards. Also it is open source and without additional development costs allowing us to reduce the necessary time and costs for managing the communication between microcontroller and WiFi module. In fact the Microchip certified TCP/IP stack is already loaded in the microprocessor and so you don't need to set and load the data relative to $\mathrm{WiFi}$ communication protocol. As shown in figure 7, to FlyPort WiFi module are connected:

- Power Supply Circuit for providing DC supply voltage to the FlyPort WiFi module and DALI interface circuit.

- Circuital section of the digital inputs for connection to presence and brightness sensors or to any buttons.

- Circuital section of analog inputs for connecting household appliances or lighting systems in order to control their electrical consumption or to connect the inverter's outputs of a PV plant for monitoring the energy's production.

- Circuital section of the digital outputs for allowing the command (turn on/off) of the connected devices.

- DALI interface circuit for managing and driving lighting facilities (e.g LEDs) that use this communication protocol.

- Wireless ZigBee and $\mathrm{WiFi}$ modules respectively for communication with other network's devices and for allowing remote control by internet network.

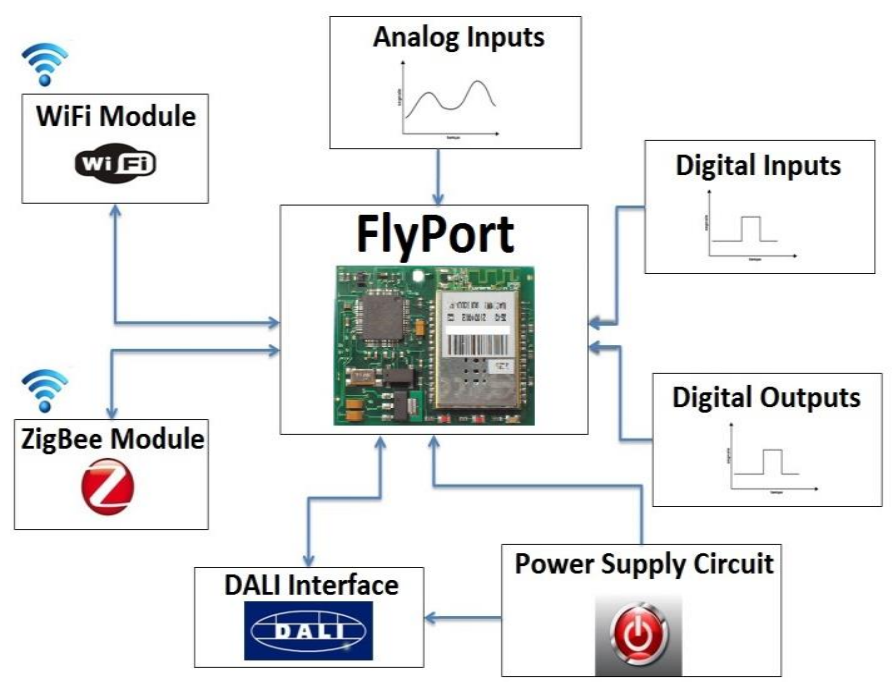

Fig. 7. Block diagram of realized monitoring/driving system with Open Picus FlyPort module connected to other functional blocks. 
In the figures 8 and 9 are shown the Open Picus FlyPort board and its block diagram with Microchip 24FJ256GA106 PIC and Wi-Fi Microchip MRF24WB0MA chip. This smart board can be used in several applications (wireless sensors network, Internet of Things, data communication security and automation); in our work PIC reads data from connected sensors and the processed data are sent to a remote web server by email or to web server located in memory of the PIC itself.

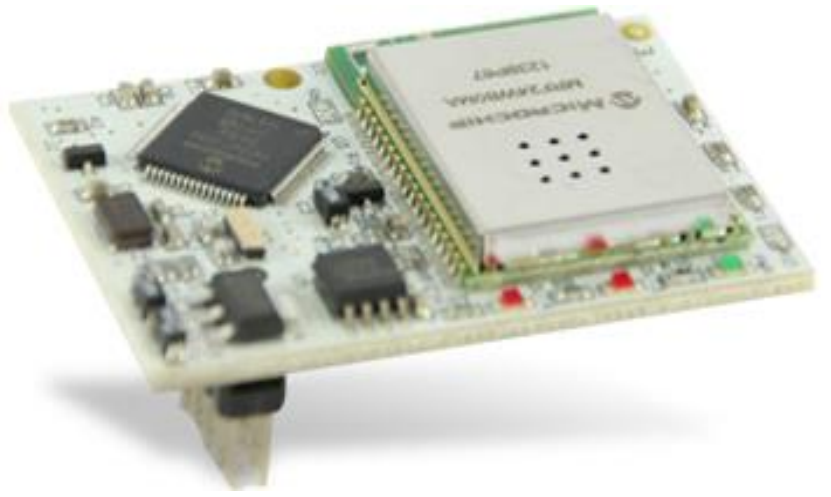

Fig. 8. Open Picus FlyPort board with embedded the WiFi module.

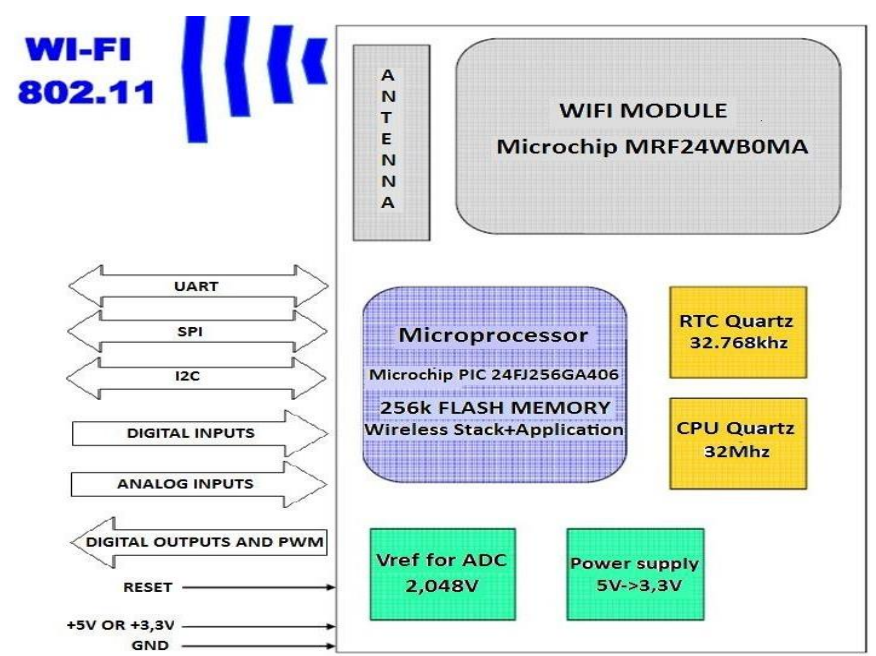

Fig. 9. Block diagram of Open Picus FlyPort board with Microchip PIC 24FJ256GA106 and WiFi Microchip MRF24WB0MA chip.

The Microchip MRF24WB0MA chip, whose block diagram is shown in figure 10 , is a low-power transceiver compatible with standard WiFi $802.11 \mathrm{~b} / \mathrm{g} / \mathrm{n}$, connected to PIC by UART interface with integrated a PCB antenna with RF distance range of $400 \mathrm{~m}$. All RF and base band components and the 802.11 medium access control (MAC) layer are embedded into WiFi module thereby creating a simple and convenient technical solution to add the WiFi connectivity to designed electronic systems.

Concerning power consumption, a maximum current of $154 \mathrm{~mA}$ is required in data transmission phase, that is reduced to $85 \mathrm{~mA}$ in reception operation. Instead during standby status, the absorbed current values are very low up to $250 \mu \mathrm{A}$ in sleep modality and $0,1 \mu \mathrm{A}$ in hibernate status.

The Open Picus FlyPort board is powered by DC $5 \mathrm{~V}$ supply voltage while PIC and embedded WiFi module need power supply of 3.3V obtained by using LT1117 voltage

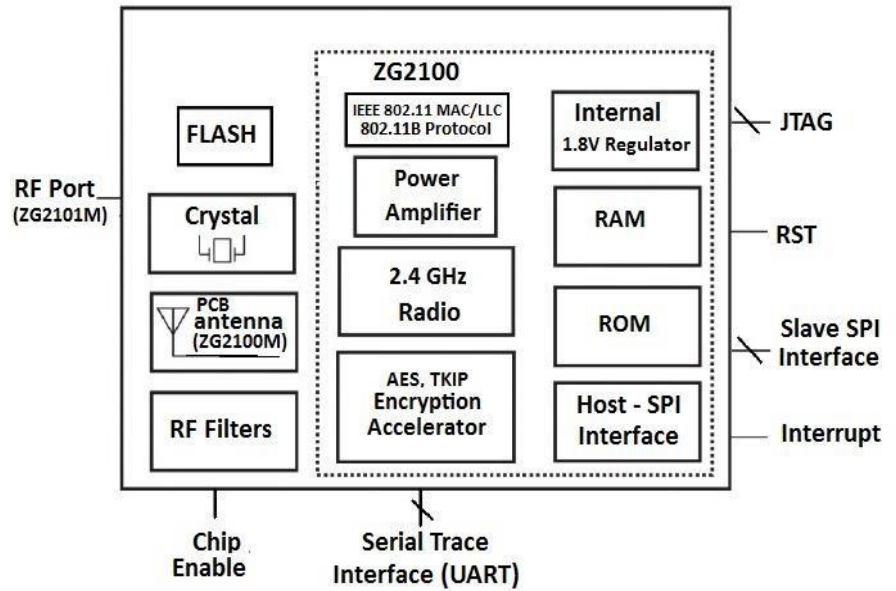

Fig. 10. Block diagram of the WiFi Microchip MRF24WB0MA module embedded into the Open Picus FlyPort board.

regulator. Depending on the application, communication with external devices is done through different interfaces (UART, SPI, I2C) and by I/O pins. As shown in figure 9, two quartz oscillators with $32 \mathrm{MHz}$ (CPU quartz) and $32.768 \mathrm{kHz}$ (Real Time Clock - RTC quartz) frequencies are used, the first to synchronize PIC's operations while the second for counting the real time. With regard to analog inputs, PIC has embedded a 10-bit ADC with a $2.048 \mathrm{~V}$ reference voltage $\mathrm{V}_{\text {REF }}$, provided by ADR5040 Precision Micropower Shunt Voltage Reference, in order to obtain a A/D conversion's resolution of $2 \mathrm{mV}$.

\section{CiRCUITAL SCHEMATICS RELATIVE TO SUPPly VOLTAGE, DIGITAL AND ANALOG INPUTS AND DIGITAL OUTPUTS.}

In this section, the circuital schematics of functional blocks connected to Fly-Port board are described in detail.

\section{A. Circuital Schematic for DC Supply Voltage}

For powering the FlyPort board and other blocks that need DC supply voltage, two circuital solutions were implemented. The first uses the MYRRA 47236 ac/DC converter that provides two DC output voltages $(5 \mathrm{~V}$ and $12 \mathrm{~V}$ this latter to supply DALI interface circuit). The second solution is based on ST Application Note AN2544 that uses ViPer22A IC device (U23 in figure 11) in buck configuration; the latter solution is cheaper not being necessary the ac/DC converter and optoisolator but does not have the galvanic isolation.

The circuit shown in Figure 11 converts the AC input (230V rms voltage, freq. $=50 / 60 \mathrm{~Hz}$ ) in DC output voltage with values of $5 \mathrm{~V}, 12 \mathrm{~V}$ or $16 \mathrm{~V}$ and a $350 \mathrm{~mA}$ available current. The $\mathrm{D}_{7}$ diode rectifies the ac input sinusoidal voltage (Phase) while $\mathrm{C}_{18}, \mathrm{C}_{19}$ and $\mathrm{L}_{1}$ components form a filter for reducing the noise from electromagnetic interference. $\mathrm{R}_{\mathbf{2 3}}$ resistance has two purposes, to limit the inrush current and to act as a fuse in case of failure. The voltage on $\mathrm{C}_{19}$ capacitor is provided by 4-pin drain of ViPer22 IC, the internal current generator provides $1 \mathrm{~mA}$ on pin 4 (VDD) for loading $\mathrm{C}_{14}$; when voltage on VDD pin reaches the value of $14.5 \mathrm{~V}$ then current generator turns off and the ViPer22 IC devices starts to pulse.

The control loop by means of $\mathrm{D}_{11}, \mathrm{C}_{17}$ and $\mathrm{D}_{9}$ produces an output voltage of $12 \mathrm{~V}$ and a $350 \mathrm{~mA}$ current. For obtaining 
16V DC output value, D9 and $\mathrm{C}_{17}$ components are removed and $\mathrm{D}_{11}$ Zener diode $\left(\mathrm{V}_{\mathrm{Z}}=16 \mathrm{~V}\right)$ replaced with another Zener diode having $\mathrm{V}_{\mathrm{Z}}=20 \mathrm{~V}$. At last, $\mathrm{D}_{12}$ diode acts as protection against overvoltages. The $12 \mathrm{~V}$ DC output feeds DALI bus since it is necessary, for the high logic level, a voltage greater than $9.5 \mathrm{~V}$. In order to power all the rest of designed monitoring/driving system is used the IC LM7805 voltage regulator to get the $5 \mathrm{~V}$ DC supply voltage.

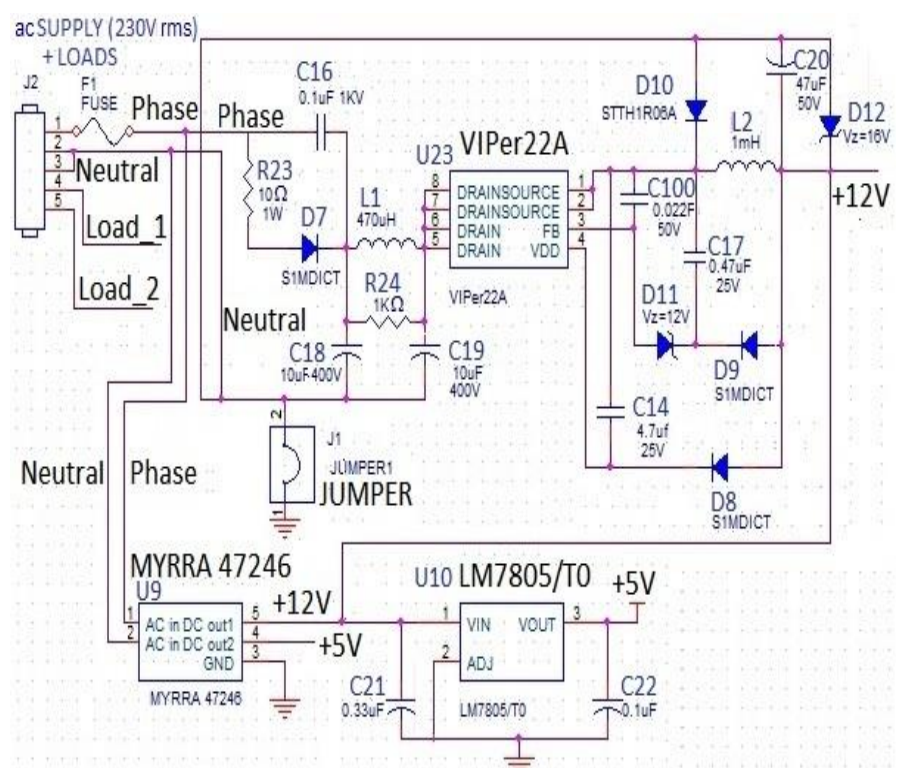

Fig. 11. Circuital schematic for DC supply voltages $(+5 \mathrm{~V},+12 \mathrm{~V}$ or $+16 \mathrm{~V}$ ) with shown the two solutions using IC MYRRA $47246 \mathrm{ac} / \mathrm{DC}$ converter or ST Application Note AN2544 with IC ViPer22A device.

\section{B. Circuital Schematic for Digital Inputs.}

Two digital inputs (PIR_1, PIR_2) are provided in the realized system whose logic level is determined by connection of presence/movement (eg PIR Passive Infrared sensor) or lighting sensors or via a button. The sensors' voltage value can be higher than $5.5 \mathrm{~V}$ tolerable from PIC's input pins (D_IN_1 and D_IN_2); for this reason, as shown in circuital solution of figure 12, by means of two opto-couplers (ISO3 and ISO4), the voltage value is shifted to a level suitable for PIC inputs (3.3 $\mathrm{V}$ for 1 logic level and $0 \mathrm{~V}$ for 0 logic level). In fact, when a low voltage is applied to the base of BJT $\mathrm{Q}_{4}$ or $\mathrm{Q}_{5}(0$ logic level to PIR input), then pnp BJT is in saturation state and the IE current, flowing through it, biases directly the optocoupler LED; therefore phototransistor is turned on in saturation and D_IN_1/D_IN_2 pins take a low logic level (voltage value = $\mathrm{V}_{\mathbf{C E}}$ of saturated phototransistor). When on PIR input is applied 1 logic level, pnp BJT is interdicted (open circuit), so opto-coupler LED is off and D_IN_1/D_IN_2 pins get a voltage of $3.3 \mathrm{~V}$ (1 logic level) via $\mathrm{R}_{19} / \mathrm{R}_{\mathbf{2 0}}$ pull-up resistors.

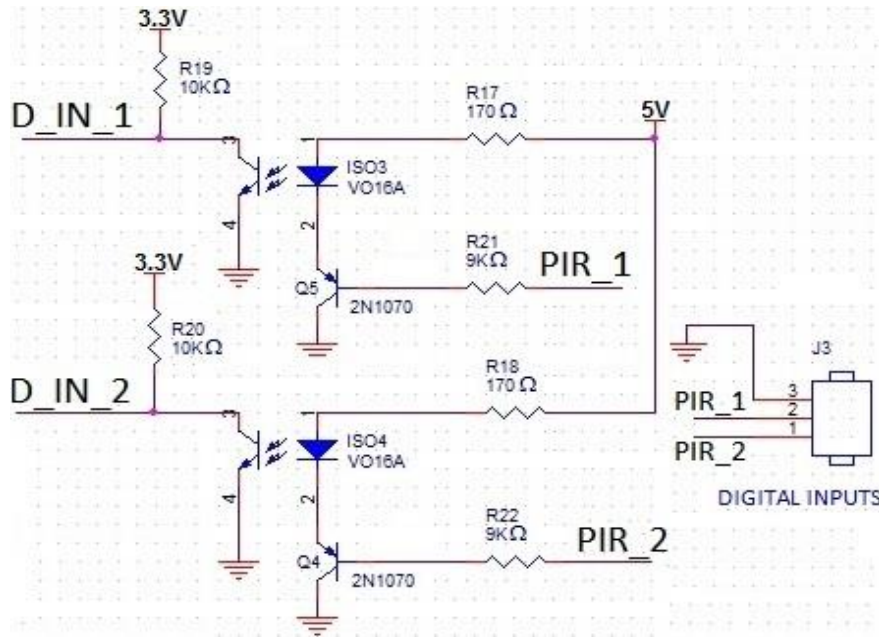

Fig. 12. Circuital schematic for digital inputs PIR_1 and PIR_2 with level adaptation circuit for obtaining D_IN_1 e D_IN_2 digital signals with voltage values suitable to be applied to PIC inputs.

\section{Circuital Schematic for Analog Inputs}

Two or more analog inputs are used to detect, by means of ACS712 current transducers, the absorbed current from household appliances or lighting facilities for monitoring their electrical consumption. In figure 13 the circuit with ACS712 Hall effect current transducer is shown; relatively to its operation mode, the four current pins $\mathbf{I} \pm$ constitute a copper conductive path inside the sensor. Ip current to be transduced generates a magnetic field detected by Hall effect internal circuit providing the output voltage Vout proportional to IP current.

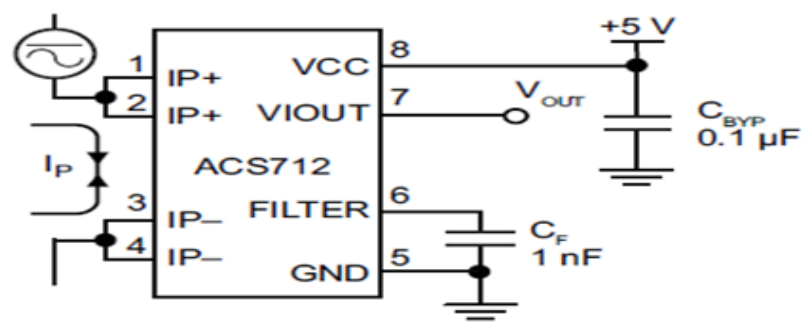

Fig. 13. Circuit relative to analog inputs for detecting, by using ACS712 current transducer, absorbed current from electrical loads.

$5 \mathrm{~V}$ supply voltage is provided by LM7805 voltage regulator of power supply section previously described, $C_{F}$ acts as filter to reduce contribution of the electrical noise. The ACS712 input/output characteristic, shown in figure 14, is linear with a positive slope for input current range [-20, +20]A; in fact the sensitivity is $100 \mathrm{mV} / \mathrm{A}$ then becoming zero if IP exceeds 20A (clamping of Vout). The output voltage Vout for zero input $\left(\mathrm{IP}_{\mathbf{P}}=0\right)$ is not 0 but approximately $\mathrm{V}_{\mathbf{C C}} / 2$, consequently Vout takes values in the following range:

$$
\left[\frac{V c c}{2}-0.1 * 20 ; \frac{V c c}{2}+0.1 * 20\right] V=[0.5,4.5] V
$$




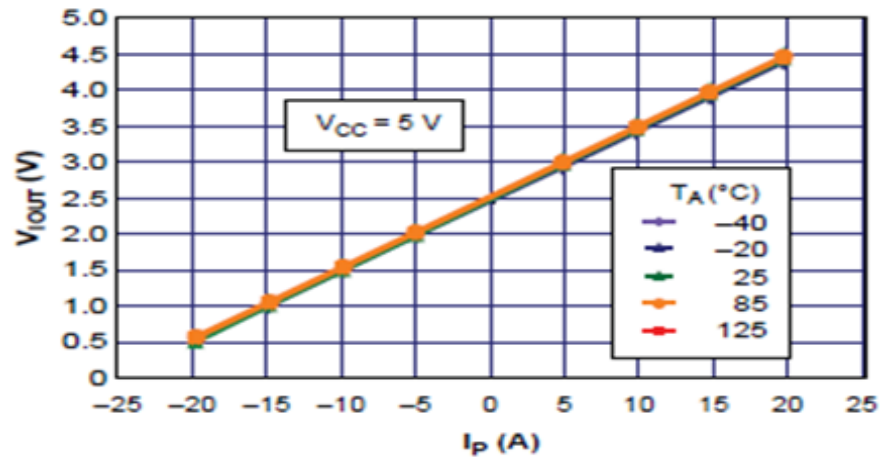

Fig. 14. Output voltage Vout as function of detected IP current for ACS712 current transducer at different operating temperatures.

Since PIC's ADC has a reference voltage VREF of $2.048 \mathrm{~V}$, output voltage Vout of ACS712 transducer is adapted by means of voltage divider (Figure 15) with resistors' values calculated by following relationship:

$$
\frac{4.5 \mathrm{~V} \times R_{12,14}}{\left(R_{12,14}+R_{11,13}\right)}=2.048 \mathrm{~V}
$$

Therefore it is obtained $R_{12,14}=0.83 R_{11,13}$ and the following values for the used resistors: $R_{12,14}=8 \mathrm{k} \Omega$ and $R_{11,13}=10 \mathrm{k} \Omega$.

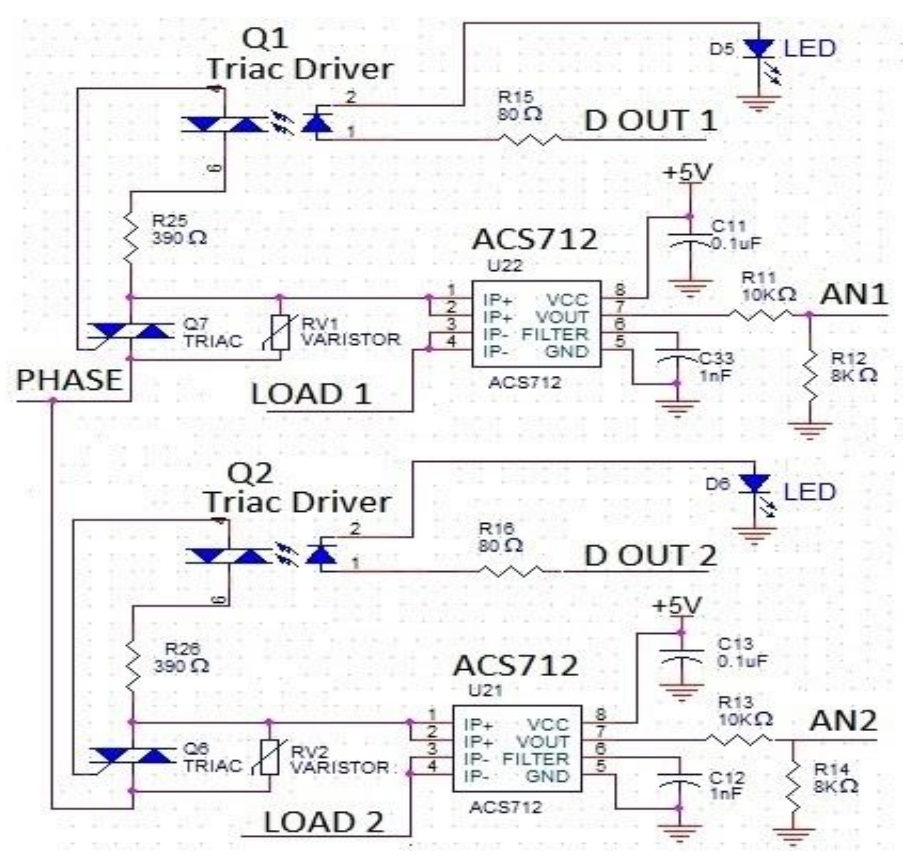

Fig. 15. Circuital schematic relative to analog inputs AN1/AN2, obtained from ACS712 current transducers and connected to PIC's ADC inputs, and relative to digital outputs D_OUT1 and D_OUT2.

\section{Circuital Schematic for Digital Outputs}

As shown in circuital schematic of figure 15, PIC's digital outputs D_OUT_1 and D_OUT_2 drive Q1 and Q2 phototriac in order to turn on/off the household electrical loads for obtaining energy savings and owner's satisfaction for domestic living's improvement. When digital outputs are set to 1 logic level, then Triac Drivers $\mathrm{Q}_{1} / \mathrm{Q}_{2}$ are activated; these last are connected to the control inputs (gate) of TRIAC Q $7 / \mathrm{Q}_{6}$ that, being switched on, allow the flow of ac current $I_{P}$ on electrical loads. The presence of $\mathrm{D}_{5} / \mathrm{D}_{6}$ LEDs allows a visual feedback when loads are activated. For limiting current (up to $5 \mathrm{~mA}$ ) flowing in phototriac LEDs and into the signaling D5 and $\mathrm{D}_{\mathbf{6}} \mathrm{LEDs}, \mathrm{R}_{\mathbf{1 5}}$ and $\mathrm{R}_{\mathbf{1 6}}$ resistors are properly sized.

\section{Circuital SECTION RELATIVE TO DALI INTERFACE FOR MANAGING AND DRIVING ADVANCED LIGHTING FACILITIES}

DALI standard allows by sending proper commands, by means of PIC, to slave lighting loads, not only the simple switching on/off of each light point, but also the ability to adjust their brightness and to manage the operating mode of lighting devices belonging to the DALI network. In order to send the commands with suitable voltage range, as seen in paragraph III, a signal level's adapting circuit is needed since, for example, voltage value relative to one logic has to be greater than $9.5 \mathrm{~V}$ value that the PIC is not able to provide.

In the circuital schematic shown in figure 16, two optocouplers are used, ISO2 on reception side and ISO1 for the transmission circuit, with the aim of adapting the signal value provided to PIC input (DALI RX side) and from the PIC's output (DALI TX side) to the DALI standard and also ensuring very fast response times. When transmitting a high logic level (DALI_TX 5V), ISO1 opto-coupler is off hence BJT Q8 doesn't conduct and bus is in the high state (idle) with DALI bus voltage equal to $12 \mathrm{~V}$. If the PIC transmitted bit is 0 logic (DALI_TX $=0 \mathrm{~V}$ ), then ISO1 opto-coupler is on state resulting a resistive path $\left(\mathrm{R}_{\mathbf{3 0}}, \mathrm{R}_{\mathbf{3 3}}\right)$ for correct polarization of BJT Q8 base (Q8 in saturation region). Thereby a current flows from $12 \mathrm{~V}$ supply voltage through diode bridge and Q8 causing a low voltage value on DALI bus (corresponding to 0 logic level).

Instead the data receiving is performed by ISO2 optocoupler, resistance $R_{31}$ and Zener diode $D_{17}$; if DALI bus is in the high logic state (it means high voltage value of DALI 1 pin), a $1 \mathrm{~mA}$ current flows through diode bridge $\mathrm{D}_{13}$ and $\mathrm{R}_{31}$ driving ISO2 opto-coupler in $\mathrm{ON}$ state and resulting a low voltage for DALI_RX pin connected to PIC input. If received bit on DALI bus is 0 logic, the corresponding voltage equal to $6.5 \mathrm{~V}$ isn't sufficient to activate ISO2 opto-coupler due to voltage drops on Zener diode D17, diode bridge and R31. If so, DALI_RX pin is floating and high voltage value guaranteed by external push-pull device. In conclusion a high level on DALI bus $(\mathrm{RX}$ bit = 1) generates a low voltage value of DALI_RX pin, while a low level on bus $(\mathrm{RX}$ bit $=0)$ generates a high signal on DALI_RX for next connection to PIC's input.

\section{PCB PROTOTYPE REALIZATION AND DESIGN OF WEB PAGE FOR REMOTE CONTROL FROM INTERNET NETWORK.}

The designed and realized CS120 Printed Circuit Board (PCB), relative to all circuital schematics described in paragraphs $\mathrm{V}$ and VI, is shown in figure 17a (top side) and 17b (bottom side with Open Picus FlyPort board embedded by using proper connector). The PCB, sized to be positioned within a container $8.5 \times 15.8 \mathrm{~cm}$, includes three different types 


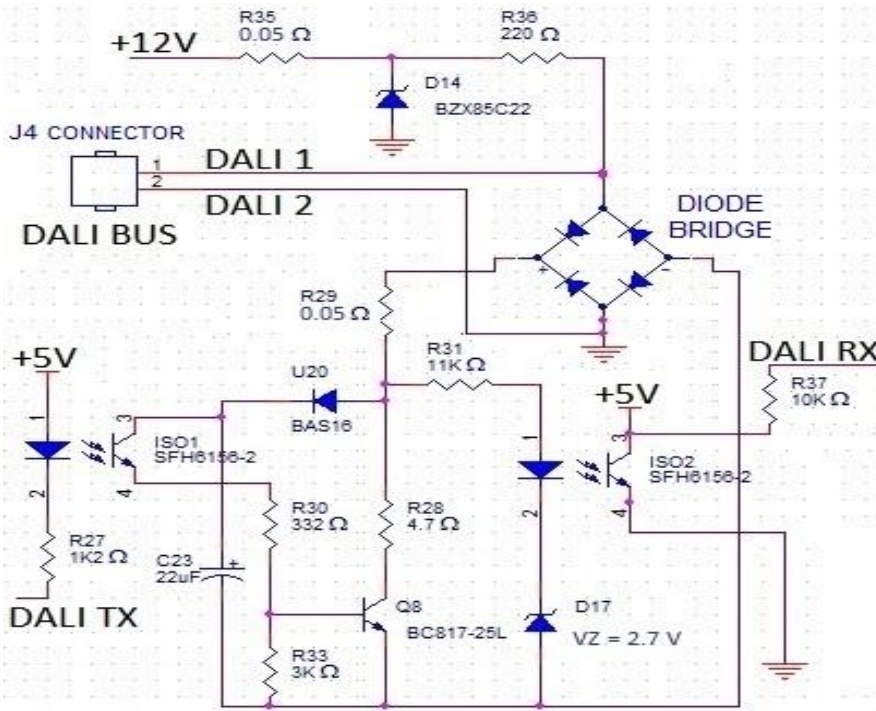

Fig. 16. Circuital schematic relative to DALI interface for adapting signals' value in the proper DALI-standard voltage range.

of electrical paths separated from each other; the conductive tracks relative to $230 \mathrm{~V}$ ac power supply, those related to DC supply voltages $(12 \mathrm{~V}, 5 \mathrm{~V}$ and $3.3 \mathrm{~V})$ and those for connecting between them all SMT and through-hole used devices. Due to different current values and lack of space for placing all used devices, the width of conductive paths relative to the ac $230 \mathrm{~V}$ supply is $1 \mathrm{~mm}$, of those related to DC voltages is $0.22 \mathrm{~mm}$ and for copper tracks relative to remaining components, $0.15 \mathrm{~mm}$.
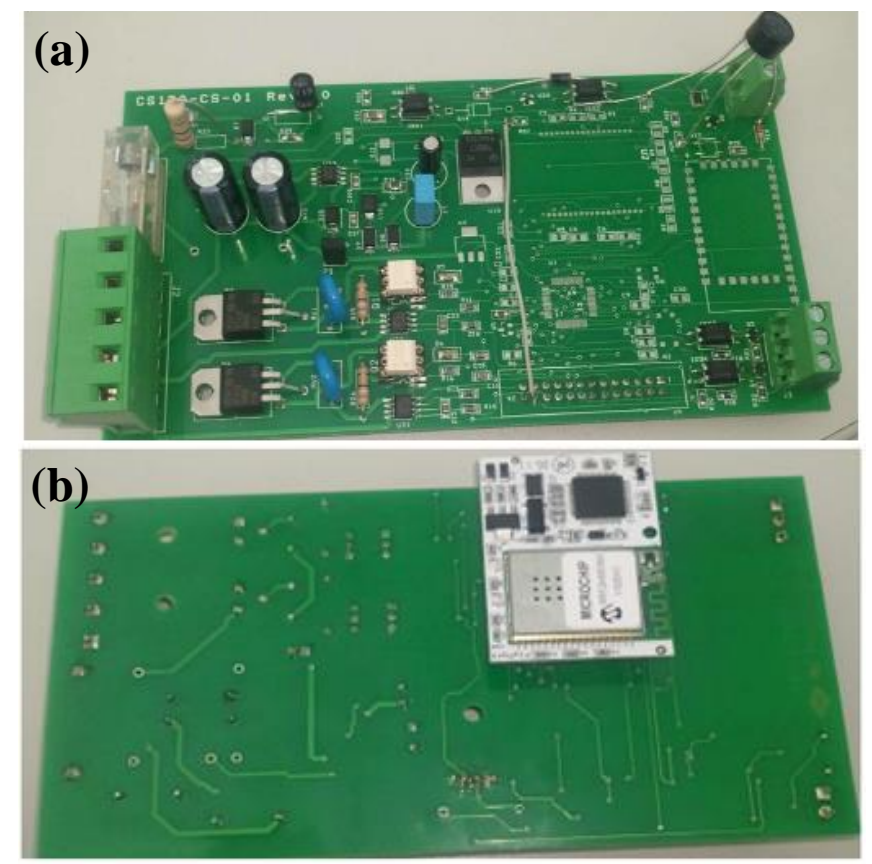

Fig. 17. Images of realized CS120 Printed Circuit Board (PCB); top side with mounted SMT and through hole components (a) and bottom side with conductive tracks and Open Picus FlyPort board embedded by using proper connector (Figure 17b).

All required components were positioned on top side except FlyPort board's connector and Myrra ac/DC converter; related to figure $17 \mathrm{a}$, the left part is dedicated to conductive tracks and components powered with $230 \mathrm{~V}$ ac supply instead the PCB's right side includes components supplied with DC voltage (not yet mounted on board in the shown picture).

The realized system uses two wireless modules, the ZigBee one makes the monitoring unit a network coordinator whereas the WiFi module allows monitoring and managing of the household electrical consumptions by connecting to web page from PC, tablet or smartphone. However in this first prototype, ZigBee module wasn't mounted on realized PCB and then the functional tests concerned WiFi monitoring and driving of connected loads by using properly created web page of which realization steps and main features will be now presented.

The Flyport web server, not different from a conventional web server, plays the server role when a client browser requires the webpage in order to view its files. The difference lies in the hardware architecture, since an embedded device, not being a professional server, has a memory, bandwidth and power computing limited; for that reason, the sent files have to be as small as possible due to reduced memory within the PIC. For writing and following loading the software into PIC's program memory, the powerful program OpenPicus IDE v. 2.2 was used.

Open-Picus FlyPort board is a stand-alone system; it incorporates the TCP/IP stack to control WiFi module and can be programmed in order to make proper actions as devices' control, reading of digital and analog inputs, communication with external devices through UART, I2C or SPI serial mode.

In the figures 18 and 19 are shown two screenshots of web page realized with dedicated NVU software; two buttons are used to turn on/off the electrical loads connected to CS120 monitoring/driving board while two LEDs display loads' state (on/off) and other two LEDs the logic level of digital inputs. By processing of absorbed current's data, real-time calculation and viewing of the instantaneous active power (W) and total consumed energy (Wh) from monitored loads (in our laboratory tests, traditional lamps o LEDs) are performed (see figure 18). Also by means of web page, the user has the ability to drive and monitor advanced lighting facilities (e.g LEDs) through DALI protocol commands (as shown figure 19).

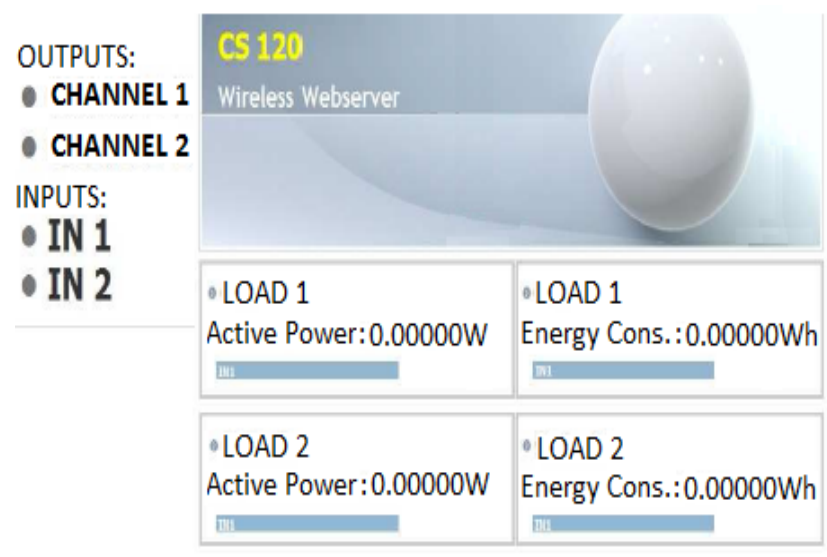

Fig. 18. Screenshot of realized web page in order to switch on/off the connected electrical loads and view, after current data processing, instantaneous active power (W) and overall consumed energy (Wh). 


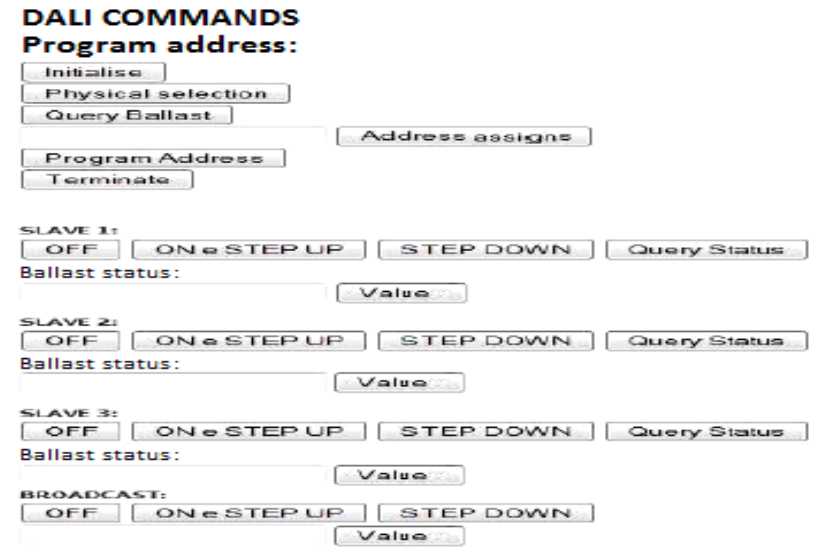

Fig. 19. Web page's screenshot related to DALI commands for driving and monitoring right operation and consumption of slave DALI lamps.

After programming Flyport module with user firmware, connector for supplying CS120 board was inserted (signaling green LED turned on). After few seconds, a red LED lights up in order to indicate the network creation from Flyport module.

Clicking on PC the icon of available wireless connections, Flyport-Net network appears (as shown in figure 20) and clicking on "Connect" a WiFi connection between PC (tablet or smartphone) and the realized CS120 PCB is established for allowing remote control by internet network. Then typing in address bar of a browser (in our case Mozilla FireFox) the IP address of FlyPort module (192.168.1.115), the realized web page will appear on browser's screen (as shown in figure 20).

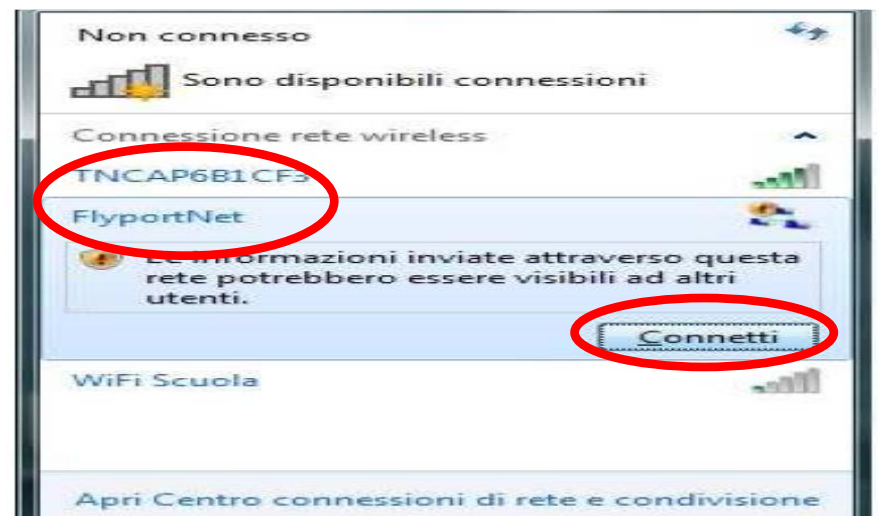

Fig. 20. Window of wireless available connections with FlyportNet; clicking on "Connect", a WiFi connection, between PC and CS120 $\mathrm{PCB}$, is established for allowing remote control by internet network.

\section{ELECTRICAL TESTING OF MONITORING DRIVING SYSTEM AND EXPERIMENTAL RESULTS}

To verify correct operation of whole implemented system, several tests were carried out each time building a proper experimental setup. In the first functional test, it was verified the managing, through the web page, of digital outputs and analog inputs; a Cascade transformer $230 \mathrm{~V}_{\mathrm{AC}}-24 \mathrm{~V}_{\mathrm{AC}}$ and a 40W incandescent lamp were connected to the realized CS120 PCB as shown in the circuital schematic of figure 21.

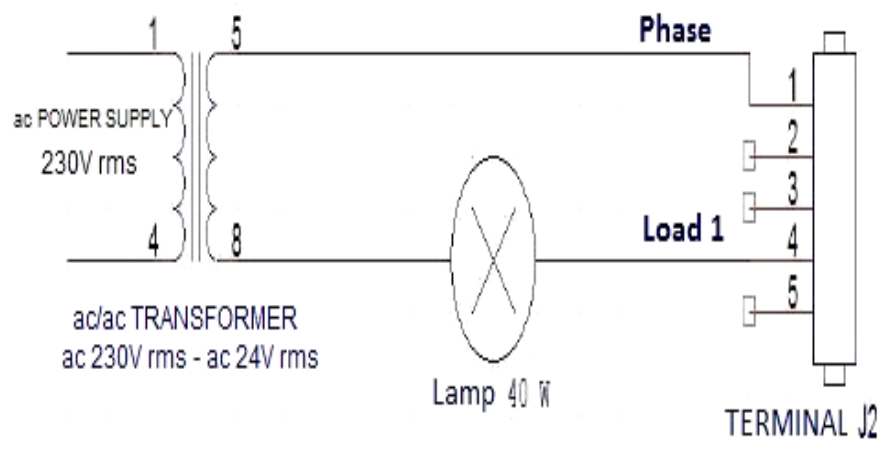

Fig. 21. Circuital schematic relative to first electrical test about managing, via the web page, of digital outputs and analog inputs.

After opening the web page, by clicking on "channel 1", the lamp connected to $\mathrm{J}_{4}$ connector is turned on (also LED D5 on CS120 PCB as shown in figure 22). Then the ACS712 current transducer provides, to PIC's analog input AN1, the values of absorbed current for calculating and viewing on web page of active power (W) and energy (Wh) consumed from load lamp for each minute. In figure 23, the flow chart concerning executed operations and calculations in this first test is shown. Clicking again on "channel 1" after an hour of monitoring (see figure 18), the $40 \mathrm{~W}$ lamp is turned off by stopping energy counting to value of $38.30 \mathrm{Wh}$ with an error of $4.25 \%$. The same test was carried out by connecting the $40 \mathrm{~W}$ lamp on second channel (pin 5 of $\mathrm{J}_{\mathbf{2}}$ terminal) and clicking "channel 2" on the web page. In this case, after an hour, the calculated value of used energy was $38.20 \mathrm{Wh}$ with an error of $4.5 \%$. The obtained results are consistent with a $4 \%$ measuring error of ACS current transducer as reported in its datasheet.

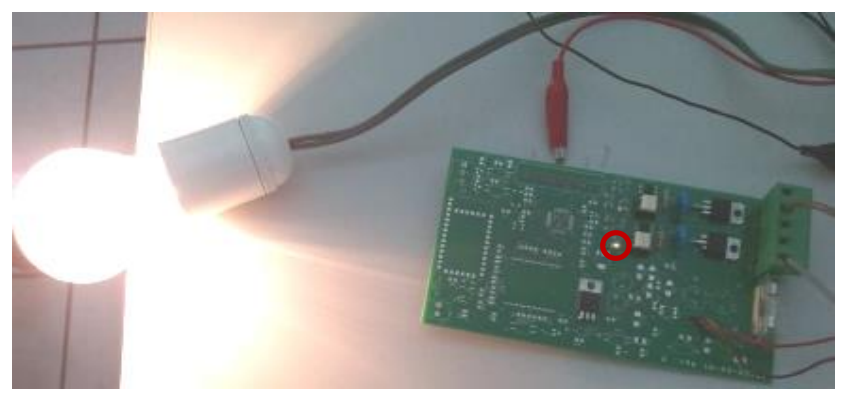

Fig. 22. 40W incandescent lamp connected to driving/monitoring board (D5 LED ON in the red circle) during first functional test.

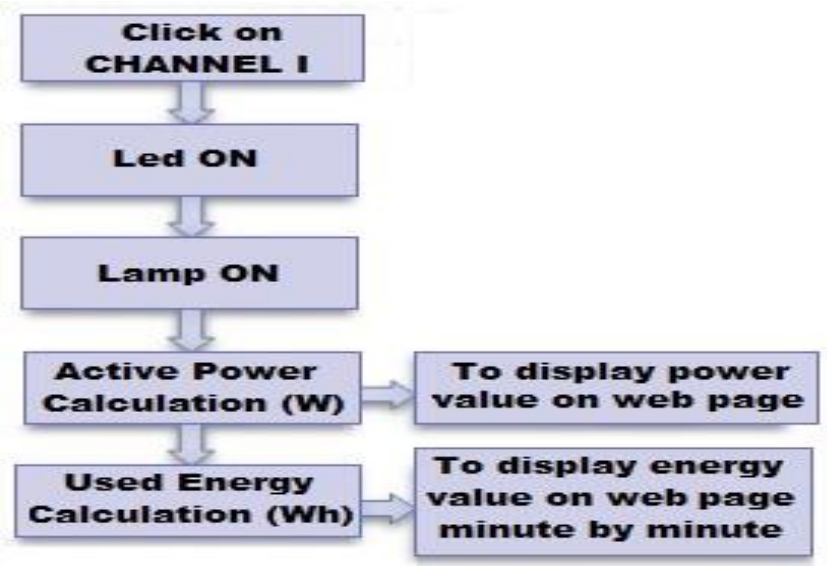

Fig. 23. Flow-chart concerning the executed operations and calculations in this first functional test. 
In the second functional test, it was tested the storage of detected/calculated data into an EEPROM device connected to PIC's I2C interface (pins 1-3 of $\mathrm{J}_{9}$ connector). The realized firmware counts the used energy every minute from monitored lamp and, in addition to view it on web page, store it in the EEPROM device. Thus, in case of power failure, the energy calculation will restart from last saved value so taking memory of lamp's consumed energy in all its operational life. In figure 24 , the flow chart relative to operations and calculations, as above described, is shown. The performed test was successful verifying the proper operation of EEPROM device; after power removal, the counting has stopped and started again, when power restored, from last value stored in the memory.

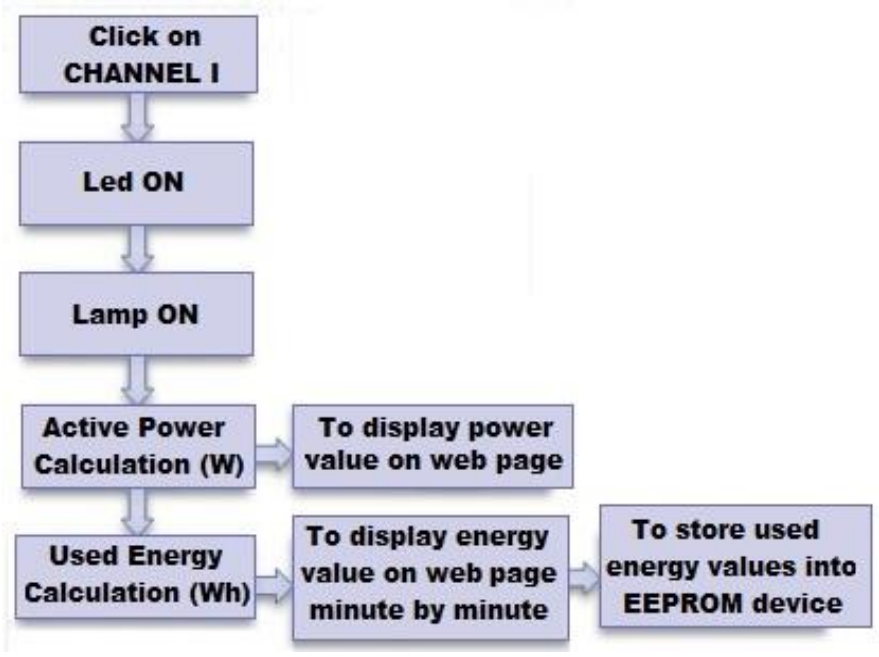

Fig. 24. Flow-chart relative to operations and calculations of the second test for verifying proper functioning of EEPROM device.

In the third functional test, it was verified the proper functioning of PCB circuital section relative to digital inputs (shown in figure 25). The ignition of load lamps connected to $\mathrm{J}_{\mathbf{4}}$ terminal (and counting of dissipated energy) can also be activated via digital inputs of CS120 PCB (pins 2 and 3 of $\mathbf{J}_{\mathbf{3}}$ terminal, see figure 12). By applying 5Volt (1 logic level) to external pin 2 (3), PIC's digital output D_OUT_1 (D_OUT_2) is activated for turning on the lighting system or other household electrical loads (on web page, the LED relative to digital input is switched on). Instead by applying 0 logic level to digital input, by using presence or lighting sensors, the lamps are switched off for obtaining energy savings.

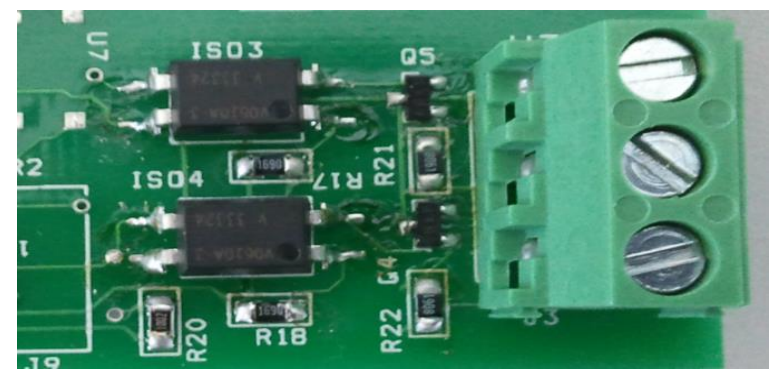

Fig. 25. CS120 PCB's section related to the digital inputs (top side).

In the subsequent functional test, the proper operation of DALI interface's circuit was verified by using 3 LED strips (shown in figure 26a), each one driven by a slave ballast
(MEANWELL LCM 60DA shown in figure 26b) connected to DALI bus ( $\mathrm{J}_{\mathbf{4}}$ terminal of CS120 PCB) as reported in adapting circuit of DALI signal level (figure 16).
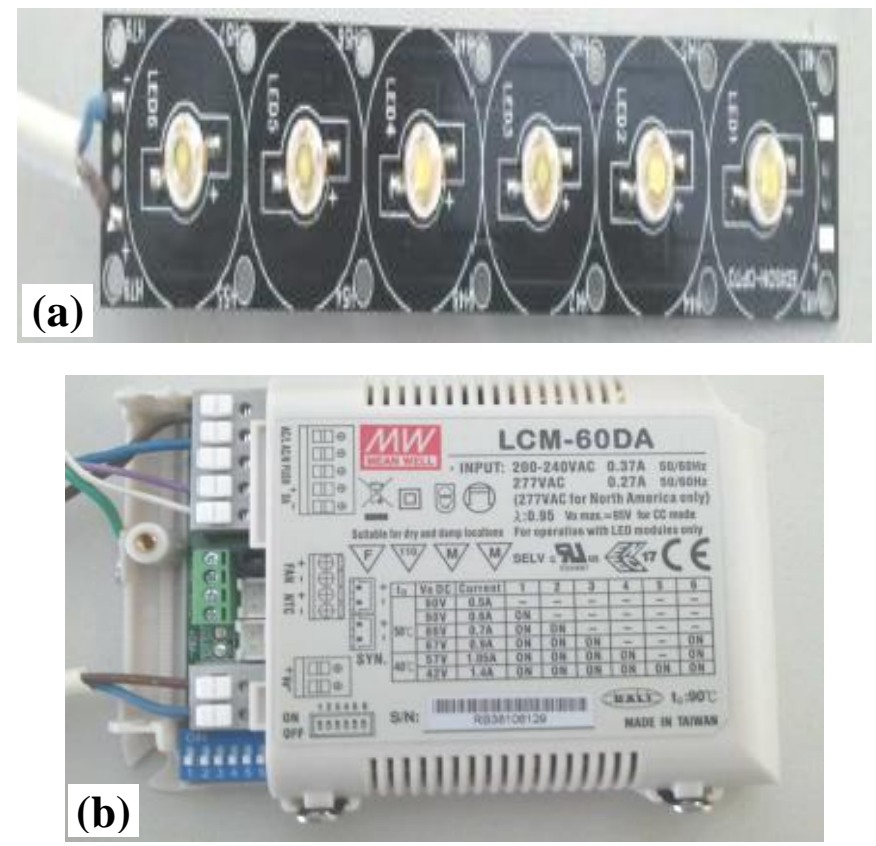

Fig. 26. LED stripline used for verifying proper operation of DALI interface's circuit (a) and MEANWELL LCM 60DA ballast, connected to DALI bus, in order to properly drive power LEDs (b).

For verifying proper functioning of CS120 driving board with master-role in the DALI network, a forward frame with Query Status command was sent from master unit to Meanwell ballast slave requiring its response. The oscilloscope probe was connected on DALI bus $\left(\mathrm{J}_{\mathbf{4}}\right.$ terminal of the PCB) in order to detect the bit strings sent to slave ballast and then from the latter to the CS120 master unit. In figure 27 the first bits string sent to slave ballast is shown followed, after a time interval of $5.2 \mathrm{~ms}$, from the string sent from slave ballast to CS120 master control unit (backward frame).

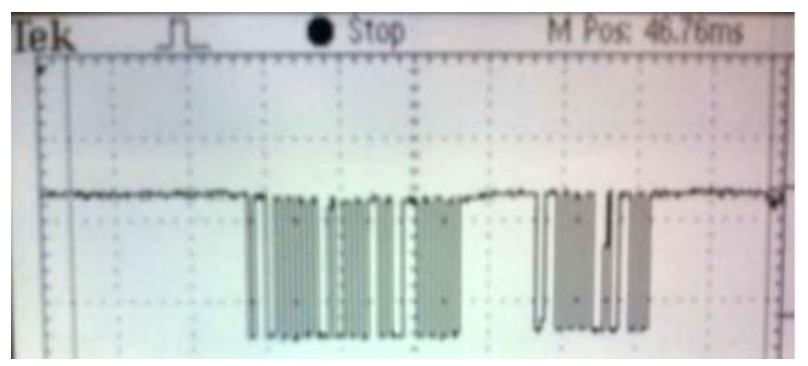

Fig. 27. Oscilloscope detection of bit strings on DALI bus; the first sent to slave ballast and the next one sent from slave ballast to CS120 master control unit.

The single bit duration is $419.65 \mu$ s, that of whole forward frame is $15,84 \mathrm{~ms}$; the voltage values relative to 1 and 0 logic levels are respectively 12.7 Volt and 2.9 Volt as required by DALI standard and confirming correct operation of signals level's adapting circuit. For obtaining a correct synchronization of data transmission, in the firmware, PIC's timers were used, in particular timer 2 for sending of forward frame and timer 4 for the reception of backward frame. 
LED lighting strips (figure 26a) were programmed with Physical Selection mode; on the web page shown in figure 19, a click on button for ballast address assignment allows sending of DALI commands to respective slave identified with unique address (001000 for slave 1, 000100 for slave 2 and 000011 for third slave ballast).

For driving each LED strip, in web page some buttons are present: OFF (for switching off LEDs), ON e STEP UP (to turn on and raise the lamp's brightness level by one step), STEP $D O W N$ (to reduce the brightness level) and QUERY BALLAST (in order to ask slave status and, if operating properly, displayed text on web page will be "OK" otherwise "FAIL"). Also the user, remotely through web page, can set a specific value of brightness, arc power from 0 to 255 , for adjusting lamp's light intensity according to user needs.

In the experimental tests, three ballasts were controlled with different arc power values; as can be seen from images of figure 28, performed tests were successful being able to drive, from web page, LED lamps with different and specified light intensity.
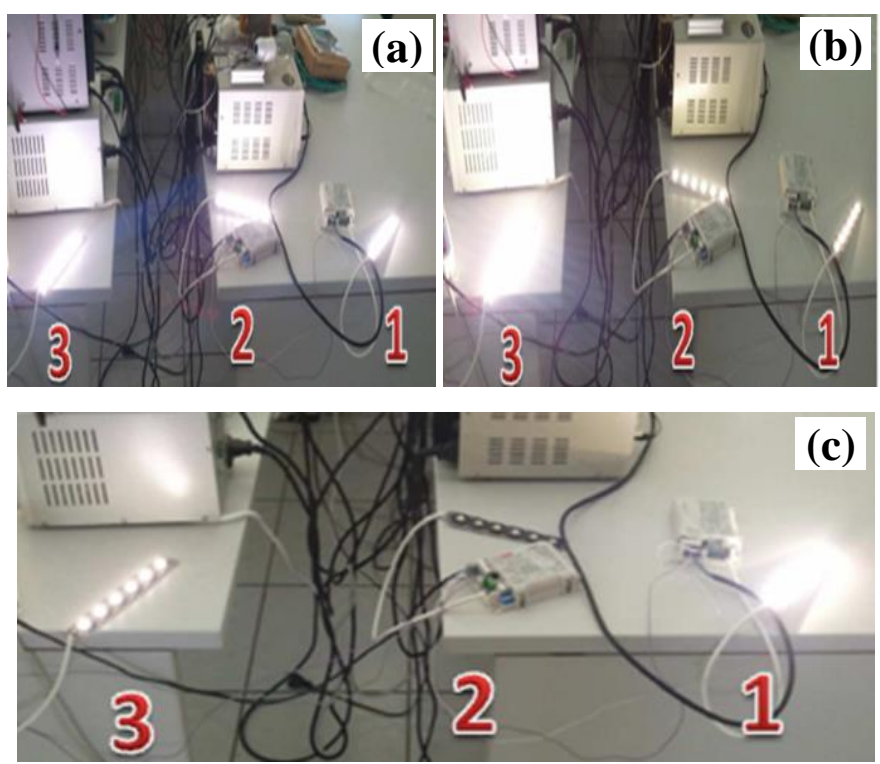

Fig. 28. For verifying correct sending, by using web page, of DALI commands to slave ballasts, specified tests were carried out driving LED lamps with different Arc Power levels. First experimental test with all three LED strips driven for maximum light intensity (Arc Power level 255) (figure a). Second test with light intensity level for slave 1 of 10, slave 2 with level 179 and slave 3 with level 250 (figure b) and lastly third test with Arc Power levels equal to 234, 0 (turned off) and 25 respectively for slave ballasts 1, 2 and 3 (figure c).

\section{CONCLUDING REMARKS}

In this work, we have realized a hardware smart apparatus with PIC-based software for wireless managing and remotely control of household electric facilities based on both ZigBee and $\mathrm{WiFi}$ wireless technologies. The design electronic system is able to monitor the electricity consumption of domestic electrical loads (e.g. household appliances, lighting facilities based on LEDs or traditional incandescent lamps) reporting the detected data consumption on a web page properly realized for remote control.

By means of web page, the user can remotely turn on/off the managed household facilities for obtaining energy savings and/or owner's satisfaction due to domestic living's improvement. Moreover remotely by using web page, the monitoring system allows by sending, through the PIC, specific DALI-standard commands to slave lighting devices, not only their switching on/off but also the ability to monitor the operating status and to adjust their brightness level as function of natural light intensity (detected by light sensor) or presence inside the room (detected by presence sensor).

All functional tests, carried out on realized CS120 PCB by using proper laboratory experimental setups (described in detail in paragraph VIII), have provided positive results allowing, for future developments, the use of developed monitoring/driving unit of household facilities in real house's electrical plant.

Within a context of green economy and energy saving with regard to the household electricity consumptions, there are many possible applications for the realized monitoring/driving apparatus, for example the monitoring of energy production from a photovoltaic plant. In this case, ACS712 current transducers will measure current and voltage values produced from PV strings and then, after PIC-based data processing, the calculated values relative to produced instantaneous power (W) and energy at a given time interval (KWh) are displayed on the page web allowing remote control of the PV plant.

\section{REFERENCES}

[1] P.Visconti, G.Cavalera, "Intelligent System for Monitoring and Control of Photovoltaic Plants and for Optimization of Solar Energy Production" IEEE Conf. Proc. of Int. Conf. Environment and Electrical Engineering EEEIC 2015, pp. 1933-1938, DOI: 10.1109/EEEIC.2015.7165468 (2015).

[2] P.Visconti, P.Costantini, G.Cavalera, "Design of electronic programmable board with user-friendly touch screen interface for management and control of thermosolar plant parameters" - IEEE Conf. Proc. of Int. Conf. Environment and Electrical Engineering (EEEIC 2015), pp.284-289 -DOI: 10.1109/EEEIC.2015.7165553.

[3] J. Han, C. Choi, WanKi Park, Ilwoo Lee, "Smart Home energy management system including renewable energy based on ZigBee and PLC" , IEEE Transactions on Consumer Electronics, Vol.60 (Issue 2) pp.198-202, DOI:10.1109/TCE.2014.6851994 (2014).

[4] P.Visconti, P.Costantini, G.Cavalera "Thermo-solar plant managing and monitoring by electronic programmable device with touch-screen interface and local or remote control possibility", Int. J. of Engineering Research \& Technology IJERT, Vol.4, pp.1019-1026, DOI:10.17577/IJERTV4IS041041 (2015).

[5] K. Gill, Shuang Hua Yang, Fang Yao, Xin Lu, "A ZigBee based Home Automation System" - IEEE Transactions on Consumer Electronics, Vol. 55 - Issue 2, pp. 422 430, DOI: 10.1109/TCE.2009.5174403 (2009).

[6] M. Magno, T. Polonelli, L. Benini, E. Popovici "A low cost, highly scalable wireless sensor network solution to achieve smart LED light control for green buildings" - IEEE Sensors Journal, Vol. 15,pp.2963-2973, DOI:10.1109/JSEN.2014.2383996 (2015). 
[7] O. Kaplan, F. Issi, M. Ersan ; “A high efficient driver design for LED lighting system" IEEE Proc. of Fourth Int. Conf. on Power Engineering, Energy and Electrical Drives (POWERENG 2013), pp.1548 -1552, DOI:10.1109/PowerEng.2013.6635846 (2013).

[8] A. Kar, A. Kar ; "Advancements in solid state lighting technology for smart and energy efficient illumination system design" IEEE Proceed. of Intern. Conference on Green Computing Communication and Electrical Engineering (ICGCCEE 2014), pp. 1-6, DOI: 10.1109/ICGCCEE.2014.6922395 (2014).

[9] A.M. Dollah, S.A.M. Al Junid, Z. Othman, F.N. Osman, K.K.M. Shariff "Energy-Efficient Light Driver Controller Using Complex Programmable Logic", IEEE Proc. of 2013 Conf. on Systems, Process \& Control (ICSPC 2013), pp. 262 - 267, DOI: 10.1109/SPC.2013.6735144 (2013).

[10] S.C. Wang, Y.H. Liu, Y.L. Chen, J.Y. Chen "Development of DALI-based electronic ballast with energy saving control for ultraviolet lamps" IEEE Proc. 8th Conf. on Industrial Informatics, pp.214-219, DOI: 10.1109/INDIN.2010.5549427 (2010).

[11] F. Domingo Perez, A. Gil-de-Castro; J.M. Flores Arias, F.J. Bellido-Outeirino, A. Moreno-Munoz, "Lighting control system based on DALI and wireless sensor networks", 2012 IEEE Innovative Smart Grid Technologies (ISGT), pp. 1-6, DOI: 10.1109/ISGT.2012.6175666 (2012).

[12] J. Byun, I. Hong, B. Lee, S. Park, "Intelligent Household LED Lighting System Considering Energy Efficiency and User Satisfaction", IEEE Transactions on Consumer Electronics, Vol. 59 - No.1, pp. 70-76, DOI: 10.1109/TCE.2013.6490243 (2013).

[13] D. Evans, "The internet of things: How the next evolution of the internet is changing everything" CISCO white paper, (2011).

[14] J. Williams, "Internet of Things: Science Fiction or Business Fact? " Harvard business review - Analytic Services, sponsored by Verizon. (2014).

[15] Chakkor Saad, Baghouri Mostafa, El Ahmadi Cheikh, "Comparative performance analysis of wireless communication protocols for intelligent sensors and their applications", Int. J. of Advanced Computer Science and Applications IJACSA, Vol. 5 No. 4, pp. 76-85, DOI: 10.14569/IJACSA.2014.050413 (2014).

[16] Yepeng Ni, Fang Miao, Jianbo Liu, Jianping Chai, "Implementation of Wireless Gateway for Smart Home", Communications and Network, Vol. 5, pp. 16-20, DOI:10.4236/cn.2013.51B005 (2013).

[17] T. Obaid, H. Rashed, A. Abou-Elnour, M. Rehan, M.M. Saleh, M. Tarique, "ZigBee Technology and its application in Wireless Home Automation systems: a survey", Int. Journal of Computer Networks \& Communications - IJCNC, Vol.6 - No.4, pp. 115131, DOI : 10.5121/ijcnc.2014.6411 (2014).

[18] Huang Jinhui, Xue ZiYu, Xu Xu, Tian Siyang, "ZigBee-based Intelligent Home Furnishing", Int. Journal of Smart Home, Vol. 9, No. 1, pp. 61-68, DOI : 10.14257/ijsh.2015.9.1.07 (2015).

[19] Datasheet EM250 Single-Chip ZigBee/802.15.4 Solution: http://www.eet-china.com/articles/2005oct/pdf/em250_datasheet. pdf?sources=download

[20] ETRX2 ZIGBEE module Product Manual http://www.telegesis.com/download/document-centre/etrx2 _module_based_products/TG-ETRX2-PM-001-110.pdf

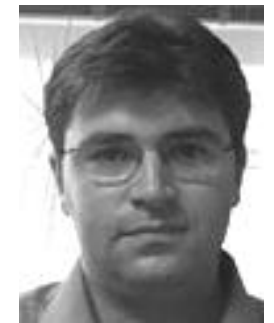

Paolo Visconti received the M.S. degree in Electronic Engineering from University of Pavia (Italy) in 1996 discussing a thesis in microelectronics and $\mathrm{PhD}$ from University of Lecce (Italy) in 2000 in the research field of photonic and electronic nano-devices. From March till to December 2000, he was visiting professor at Virginia Commonwealth University (USA) working on fabrication and characterization of optical GaN-based devices. Since 2001 he is carrying on his research and teaching activity as Assistant Professor in Electronics at the Dept. of Innovation Engineering - University of Salento (Italy) overseeing more than 60 students' research activities for B.S., M.S. and Ph.D levels. Actually his research interests include design and realization of smart PIC-based electronic boards for monitoring and dataacquisition systems. He is author or co-author of more than 100 papers published in international journals, books and conferences' proceedings.

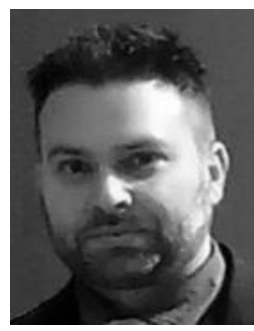

Patrizio Primiceri received the M.S. degree in Telecommunications Engineering from Salento University (Italy) in 2010 with thesis on electronic for telecommunications. In September 2010, he won a research grant at Milano-Bicocca University as microelectronic designer in collaboration with Pirelli S.p.A and in October 2012 a scholarship with Italian National Institute of Nuclear Physics; currently he is working with Salento University as electronic designer. His research interests include analog and digital circuits design, SMT Printed Circuit Board design, firmware programming for smart PIC-based data-acquisition boards.

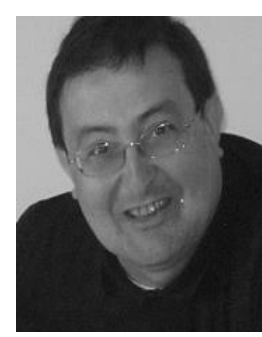

Giorgio Cavalera is currently head of Cavalera Ltd R\&D with over ten-year experience in electronic designing and developing of hardware and firmware for microcontroller-based embedded systems realized for monitoring and control applications. Particularly he is expert in the firmware's development for time-critical systems. To date he developed about 160 projects installed in thousands of complex systems. He is holder of several patents and co-author of 16 scientific publications. 